\title{
Torsion hematosalpinx concurrent with acute appendicitis
}

\author{
Vishws G. Kanabar ${ }^{1 *}$, Ravi N. Kotecha ${ }^{2}, \operatorname{Ramesh}_{\text {Thakan }}^{2}$
}

\author{
${ }^{1}$ Department of Obstetrics and Gynecology, Shubham Hospital, Phalodi, Jodhpur, Rajasthan, India \\ ${ }^{2}$ Department of General Surgery, Shubham Hospital, Phalodi, Jodhpur, Rajasthan, India
}

Received: 26 June 2021

Accepted: 27 August 2021

\section{*Correspondence:}

Dr. Vishws G. Kanabar,

E-mail: dr.v..g.kanabar@gmail.com

Copyright: (C) the author(s), publisher and licensee Medip Academy. This is an open-access article distributed under the terms of the Creative Commons Attribution Non-Commercial License, which permits unrestricted non-commercial use, distribution, and reproduction in any medium, provided the original work is properly cited.

\begin{abstract}
Isolated fallopian tube torsion is very rare which is reported to be 1 in 1.5 million women. Fallopian tube torsion is commonly associated with hydrosalpinx, hematosalpinx or paratubal cysts such as hydatids of Morgagni. Fallopian tube torsion generally presents a diagnostic dilemma because symptoms and signs mimics ovarian torsion such as acute lower abdominal pain, vomiting, tenderness on palpation but ultrasound may show normal ipsilateral ovary. Right sided adnexal torsion may be difficult to differentiate from acute appendicitis clinically. In such cases ultrasound is helpful but diagnostic laparoscopy is gold standard. We encountered a rare case of torsion hematosalpinx concurrent with acute appendicitis. Patient was presented with acute onset severe lower abdominal pain associated with vomiting and tenderness in right iliac fossa. It was diagnosed as acute tip appendicitis and right adnexal cystic mass either hydrosalpinx/hematosalpinx in ultrasonography. Laparoscopy was done and it was found to be right sided torsion hematosalpinx along with inflamed tip of appendix. Right sided salpingectomy and appendicectomy was done laparoscopically. Fallopian tube torsion should be suspected and diagnostic laparoscopy should be considered in cases of acute onset lower abdominal pain in which ovarian pathology was not found in ultrasonography, that helps in earlier intervention and even in early cases fallopian tube can be salvaged.
\end{abstract}

Keywords: Hematosalpinx, Torsion hematosalpinx, Fallopian tube torsion, Laparoscopy, Acute appendicitis

\section{INTRODUCTION}

Fallopian tube torsion, while far less common than ovarian torsion, may present in a remarkably similar fashion. Isolated fallopian tube torsion has an incidence reported to be 1 in 1.5 million in adult women. ${ }^{1}$ Tubal torsion may present as a diagnostic dilemma since the ovary itself usually appears normal on ultrasound. Thus, surgical intervention may be delayed which can lead to worsening necrosis and result in the need for resection of the affected tube. $^{2}$

Fallopian tube torsion may be associated with hydrosalpinx, hematosalpinx or paratubal cysts such as hydatids of Morgagni. The presentation of fallopian tube torsion is similar to that of ovarian torsion: abdominal or pelvic pain which may be associated with fever, nausea, or vomiting. The pain may be acute or chronic and is sometimes less well localized than that of ovarian torsion. ${ }^{3}$ Tubal ligation has also been reported as one of the predisposing factors for IFTT (Isolated Fallopian Tube Torsion). ${ }^{4}$

Clinical features of acute appendicitis may mimic that of right sided adnexal torsion such as acute lower abdominal pain, vomiting, tenderness on examination. Ultrasound helps to differentiate them. Further, Laparoscopy appears to provide an excellent mechanism for early diagnosis and treatment of fallopian tube torsion.

Occurrence of fallopian tube torsion concurrent with appendicitis is itself very rare. Tubal ectopic pregnancy is also an important differential diagnosis in such cases of acute abdominal pain with adnexal mass in reproductive age woman especially those who are tubectomised or had any other tubal surgery. 
The torsion of the fallopian tube is usually unilateral and it occurs most commonly on the right side. This may be due to the presence of the sigmoid colon on the left side (it prevents the adnexal movement) or the slow venous flow on the right side (this results in congestion). ${ }^{5,6}$

\section{Management}

Isolated tubal torsion can be managed with either detorsion or simple salpingectomy. Adnexal de-torsion has an extremely low risk of thromboembolic events. However, it should be performed as early as possible to avoid irreversible damage to the tissue. The operative approach could be conventional exploratory laparotomy or laparoscopic surgery.

Several studies of adnexal torsion have demonstrated that the color, size, and degree of edema do not correlate with necrosis and subsequent return to normal tubal or ovarian function. Immediate detorsion is always recommended, because it is unclear how long a patient has sustained torsion until irreversible damage occurs. ${ }^{7}$

Laparoscopic detorsion of the tube is the treatment of choice whenever feasible to preserve the fertility. Whenever fertility is not desired, salpingectomy should be the procedure of choice. Devi et al suggested that most of the torsions are noted in the distal two-third of the tube, and believed that the complete salpingectomy is better than partial whenever it is necessary because the proximal nonfunctional part of the tube can be a site of ectopic pregnancy or reformation of hydrosalpinx if it gets occluded and removal of only ampulla and fimbria and keeping the nonfunctional portion is of no use and may increase the risk of recurrence. ${ }^{4}$

\section{CASE REPORT}

Here we present a very rare case of torsion of hematosalpinx concurrent with acute appendicitis. Our patient was a 27 years old female, presented with acute lower abdominal pain more on right side associated with nausea and vomiting. Her obstetric history was para 3 live 3 with previous 3 cesarean section births. Her last childbirth was 3 years back. She had undergone postpartum tubal ligation along with cesarean section. Her menstrual cycles were regular. Her last menstruation was 22 days back.

On examination, her vitals were normal. On abdominal examination, there was approximately $6 \times 4 \mathrm{~cm}$ tender cystic mass felt in right lower quadrant. on per vaginal examination, uterus was anteverted, anteflexed, normal in size and shape. Cystic mass of approximately $6 \times 4 \mathrm{~cm}$ felt in right fornix which was severely tender and cervical movements were tender. Urine pregnancy test was done which was negative.

Ultrasonography revealed a thin wall dilated (max. 24.1 $\mathrm{mm}$ ) tortuous, anechoic tubular structure with incomplete thin septation seen in right adnexa suggestive of hydrosalpinx/hematosalpinx. In right iliac fossa, distal part of appendix approx. $2 \mathrm{~cm}$ long was non-compressible, non-peristaltic with increased caliber, $6.6 \mathrm{~mm}$ in diameter, retroileal location s/o distal acute appendicitis.

Patient was taken to operative room for diagnostic laparoscopy along with consent for operative intervention as required. Operative findings were as follows: (1) inflamed appendix; (2) in pelvic area there was mild hemoperitoneum; (3) there was torsion of enlarged bloodfilled fallopian tube on its own axis. Hematosalpinx was in the part of fallopian tube which was lateral to the site of tubal ligation whereas medial part of fallopian tube was apparently normal; (4) ipsilateral ovary was normal; (5) opposite side ovary and fallopian tube was normal. Decision was taken for operative intervention. We did laparoscopic appendicectomy and resection of hematosalpingeal mass. Both were sent for histopathological examination. Post-operative period was uneventful. Histopathology confirmed the findings of hematosalpinx and distal appendicitis.

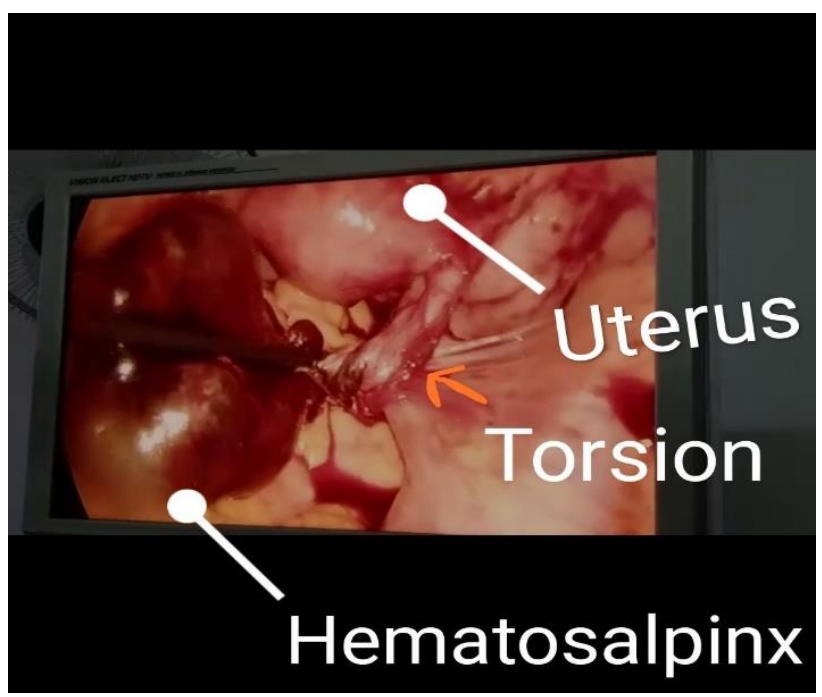

Figure 1: Right sided torsion hematosalpinx.

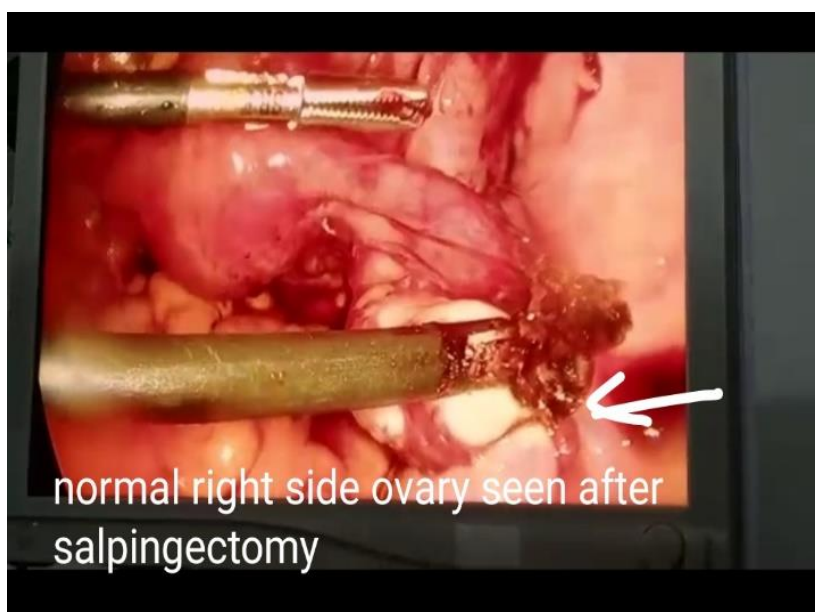

Figure 2: Right sided ovary seen after salpingectomy. 


\section{DISCUSSION}

In cases of acute abdominal or pelvic pain associated with typical symptoms and signs (nausea, vomiting, tenderness) we should always suspect adnexal torsion along with other differential diagnoses. Ultrasonography helps in the diagnosis but diagnostic laparoscopy is the gold standard. We suggest that consideration of laparoscopy as a diagnostic tool for acute onset pelvic pain in the absence of ovarian pathology may be relevant for earliest recognition and intervention.

Tubal ligation has been reported as one of the predisposing factors for IFTT. Following tubal ligation, secretions retained in the distal end may lead to formation of hydrosalpinx. This post-surgical adhesion will lead to formation of a non-mobile nidus. This combination, might predispose to torsion of tube around the nidus, although the exact pathogenesis is unknown. Hence, whenever we encounter an adnexal torsion, IFTT should be suspected in patients with a previous history of tubal ligation, with an elongated cystic mass, who have a sudden onset of abdominal pain or pelvic pain. ${ }^{4}$

Timely intervention in the form of surgery may salvage the fallopian tube in case fertility is desired and prevent other sequel of torsion even if fertility is not desired. Laparoscopy is preferred as it is minimally invasive and it also helps in diagnosis of any other pathology and operative intervention at the same time if needed.

\section{CONCLUSION}

Fallopian tube torsion is very rare condition, but it should be included in the differential diagnosis of an acute lower abdominal pain in women, especially in those with predisposing factors. An early diagnosis and an early intervention can maximize the chances of tubal salvage. Laparoscopy remains the gold standard for the same.

Funding: No funding sources

Conflict of interest: None declared

Ethical approval: Not required

\section{REFERENCES}

1. Ito F, Tatsumi H, Takahata A, Yamada S, Kusuki I, Kitawaki J. Isolated fallopian tube torsion diagnosed and treated with laparoscopic surgery: A case report. Gynecol Minim Invasive Ther. 2017;6(2):89-91.

2. Mueller C, Tomita S. Fallopian Tube Torsion as a Cause of Acute Pelvic Pain in Adolescent Females. Case Rep Pediatr. 2016;8707386.

3. Casey RK, Damle LF, Lobo V. Isolated fallopian tube torsion in pediatric and adolescent females: a retrospective review of 15 cases at a single institution. J Pediatr Adolesc Gynecol. 2013;26(3):189-92.

4. Balasubramaniam D, Duraisamy KY, Ezhilmani M, Ravi S. Isolated Fallopian Tube Torsion: A Rare Twist with a Diagnostic Challenge That May Compromise Fertility. J Hum Reprod Sci. 2020;13(2):162-7.

5. Shukla R. Isolated torsion of the hydrosalpinx: a rare presentation. Br J Radiol. 2004;77:784-6.

6. Krissi H, Orvieto R, Dicker D, Dekel A, Ben Z. Torsion of a fallopian tube following Pomeroy tubal ligation: a rare case report and review of the literature. Eur J Obstet Gynecol Reprod Biol. 1997;72(1):107-9.

7. Khairnar V, Valecha SM, Pandeeswari. Isolated twisted hematosalphinx misleading with ovarian cyst torsion. Int J Reprod Contracept Obstet Gynecol. 2019;8:1219-22.

Cite this article as: Kanabar VG, Kotecha RN, Thakan R. Torsion hematosalpinx concurrent with acute appendicitis. Int J Reprod Contracept Obstet Gynecol 2021;10:3973-5. 